\title{
Algumas questões éticas no tratamento da anorexia nervosa
}

\author{
Anorexia nervosa and treatment: some ethical considerations \\ Luiza Amélia Cabus Moreira', lrismar Reis de Oliveira²
}

\section{ANOREXIA: DEFINIÇÃO, FREQÜÊNCIA, MORTALIDADE E A RESTAURAÇÃO DO PESO}

De acordo com o DSM IV', os critérios diagnósticos para a anorexia nervosa (AN) são: a manutenção do peso corporal abaixo de $85 \%$ do esperado para a altura e a idade, grande medo de engordar (ainda que o indivíduo esteja com baixo peso), alteração da imagem corporal e amenorréia em, no mínimo, três ciclos consecutivos. Entre os transtornos alimentares (TA), representa o menos comum, porém o mais grave ${ }^{2}$. Nos pacientes com a doença, as principais causas de morte são o suicídio e os efeitos diretos do jejum ${ }^{3,4}$.

É uma patologia com relativa baixa prevalência $(0,5 \%$ a $1 \%$ em alguns estudos nos Estados Unidos), incidindo, mais comumente, em mulheres com idades entre 15 a 29 anos $^{5}$; no entanto, sua mortalidade é alta. Sullivan ${ }^{6}$, em uma metanálise englobando 42 estudos sobre mortalidade na AN estimou que a mortalidade fosse de 5,6\% por década desde o início da doença. Cerca de $20 \%$ dos afetados apresentarão doença crônica com grave comprometimento na qualidade de vida e cerca de 30\% a 40\% terão recuperação completa com o tratamento?. Em geral, os adolescentes, quanto mais precocemente forem tratados, melhor será a evolução ${ }^{8-10}$.

\section{OS PACIENTES COM ANOREXIA NERVOSA SÃO “COMPETENTES” PARA TOMAR DECISÕES?}

O critério legal para definir capacidade tem como foco o entendimento do paciente sobre sua doença e suas conseqüências, os vários tratamentos disponíveis e seus riscos e benefícios, a credibilidade nas informações para que seja tomada uma decisão por parte do indivíduo afetado pela doença, o que, obviamente, requer grande habilidade intelectual"1. A competência é o equivalente clínico da capacidade ${ }^{12}$. Os fatores relevantes para julgar competência, principalmente em pacientes psiquiátricos, englobam uma série de variáveis que não estão incluídas nas definições legais de capacidade, como a interpretação das informações por indivíduo e a influência da doença mental sobre essa ${ }^{13}$.

Os pacientes com TA não são globalmente incompetentes, mas o comprometimento de seus pensamentos, percepções, julgamentos, comportamentos e a capacidade de atender às demandas para a manutenção da vida os colocam sob risco da intervenção' ${ }^{14}$. Muitos pacientes que recusam o tratamento parecem ter a capacidade de decidir sobre o fato. Ao mesmo tempo, freqüentemente estão gravemente doentes e se beneficiarão com o tratamento se estão sob risco de vida significativo. Os pacientes com TA apresentam comorbidades (depressão, transtornos de personalidade) que podem impedi-los de procurar tratamento ${ }^{15}$.

1 Departamento de Pediatria da Universidade Federal da Bahia (UFBA).

2 Ambulatório de Transtornos Alimentares do Hospital Universitário Professor Edgard Santos (ATAH).

Endereço para correspondência: Luiza Amélia Cabus Moreira Departamento de Neuropsiquiatria FM-UFB, Av. Reitor Miguel Calmon, s/n - 40110-100 - Salvador, BA E.mail: lamelia1470@terra.com.br 
A distorção da percepção associada à inanição ${ }^{16}$ e a comorbidade com a depressão são bem conhecidas, assim como o impacto dessas sobre a competência ${ }^{17}$. No entanto, há muitos outros fatores que devem ser levados em conta quando é julgada a competência em pacientes com AN ${ }^{16,17}$.

Capacidade é o critério legal que indica ser um indivíduo capaz de tomar decisão autônoma e válida, já a competência refere-se ao conceito clínico de possuir habilidades para a tomada de decisões válidas em relação ao tratamento ${ }^{12}$. Apesar de não ser habitualmente feita clara distinção entre esses conceitos, isso seria de grande valia. A capacidade seria o conceito fixo usualmente ligado a aspectos legais, seria "a habilidade de entender e assimilar informações relevantes para a decisão em questão e assim fazer uma escolha". O conceito de competência utilizado pelos profissionais de saúde não é tão explícito em sua definição e inclui fatores adicionais de extrema importância no julgamento clínico, como consistência na expressão das decisões durante o tempo, consistência nas decisões expressas e razões subliminares por trás dessas, como desejos expressos anteriormente e a personalidade antes da doença, o estado emocional atual (especialmente se o indivíduo está sob estresse ou agitado) e seu impacto no processo da tomada de decisões, o impacto psicopatológico da doença mental nos sistemas de crenças, assim como nos sistemas de valores e desejos ${ }^{18}$.

As crenças e os valores das pessoas diagnosticadas como anoréticas, se não forem compreendidos, demonstram que os profissionais que delas cuidam podem incorrer em erros de julgamento e conseqüentemente manejo terapêutico. Por outro lado, muitos pacientes diagnosticados como portadores de AN de fato não apresentam comprometimento das suas competências e, por critérios cartesianos, podem ser julgados como competentes. Por outro lado, o paciente com competência "aparente" pode levar médicos e indivíduos ligados à Justiça a tomar decisões perigosas ou a adotarem postura paternalista para "assegurar" o melhor interesse do paciente. Para respeitar os direitos dos pacientes e proteger seus melhores interesses, a análise e a avaliação da competência na anorexia precisa ser entendida como um complexo que capture realmente os dilemas dos pacientes e dos pais.

Um outro dado que deve ser levado em conta é o fato de que a inanição leva a alterações, como demonstrado pelos estudos de neuroimagem funcional. A análise em repouso mostra hipoperfusão cerebral global ${ }^{19}$, que é mais pronunciada no singulado anterior ${ }^{20}$, região fronta ${ }^{21}$ e no lobo parietal ${ }^{19}$. Na BN, há relatos de hipometabolismo parietal ${ }^{19} \mathrm{e}$ a perda da simetria normal (que é maior no hemisfério direito em relação ao esquerdo) no fluxo no metabolismo cerebral$^{22}$. Há, também, estudos controlados utilizando imagens e formas corporais e alimentos para avaliar os sintomas relacionados aos processos cerebrais. Na AN, a perfusão dos lobos frontais (medida pela tomografia de emissão de fótons-SPECT) aumentava quando os indivíduos em estudo comiam bolo ${ }^{23}$ e com a ressonância magnética funcional, a visão de drinques calóricos levava a respostas no córtex pré-frontal, singulado anterior, córtex insular e amígdala ${ }^{24}$.

\section{O TRATAMENTO, A “COERÇÃO" E A AMBIVALÊNCIA}

Os programas de tratamento dos TA geralmente englobam equipe multidisciplinar que visa, a princípio, a restaurar o peso do paciente. A restauração do peso está associada com a melhora dos sintomas da anorexia e a melhora das funções física e cognitiva ${ }^{25}$. Essa melhora cognitiva permitirá a intervenção psicoterapêutica e psicotrópica. O peso é também fator crítico nas recaídas e reinternamentos ${ }^{26,27}$.

Dado que o objetivo, a priori, é o ganho de peso e que pacientes com anorexia apresentam como aspecto central o medo de engordar, mesmo que estejam quase morrendo em virtude da desnutrição, começa nesse momento o impasse. Quando se fala em internamento compulsório dos pacientes anoréticos, tende-se a esquecer que a resistência ao tratamento é ainda mais comum. Os quadros agudizados com risco de vida chamam a atenção dos pais, muitas vezes, para a gravidade da doença que antes era vista como "forma de chamar atenção" ou mera futilidade para aderir a padrões estéticos de magreza.

A equipe de profissionais que lida com esses pacientes geralmente não se dá conta, dada a padronização criada, que seus atos são coercitivos, se for considerado que são impostas restrições, como o tempo que o paciente pode ficar fora do leito, suas idas sob supervisão ao banheiro, o fato de terem de ingerir as calorias prescritas e que somente alimentam-se sendo supervisionadas, a proibição dos exercícios e mesmo, como alguns costumam dizer, "a prisão" (hospital) na qual estão confinadas. Uma ação que afete a autonomia do indivíduo é sempre eticamente significativa, porém não significa que seja eticamente errada. Do ponto de vista dos pacientes, a sua saúde está "boa" e julgam que as pessoas as agridem dizendo que estão doentes e provam em um discurso perfeito que estão bem na escola, que são hiperativas. No entanto, não vêem, na maioria das vezes, como estão magras.

\section{A RESISTÊNCIA AO TRATAMENTO E O INTERNAMENTO “COMPULSÓRIO"}

A resistência ao tratamento oferecida pelos pacientes com AN é o habitual. Essa se manifesta de várias formas, como na recusa de ingerir a quantidade de alimentos prescrita pela nutricionista, "truques" na hora que são pesados, remoção 
da sonda nasoenteral quando são submetidas à alimentação enteral, exercitarem-se escondidos, continuar usando laxantes e diuréticos, vomitar, entre outras. A decisão ética é um contínuo do tratamento e não apenas um fato isolado que ocorre quando a doença oferece risco imediato à vida. Ter o consentimento do paciente não deve ser visto como evento estático no tratamento, ou seja, uma vez dado está assegurado que o paciente concordará com todos os atos médicos. Várias vezes, durante a evolução do tratamento, o paciente manifesta seus medos e, por várias vezes, os aspectos da doença serão rediscutidos.

Com freqüência, o paciente com AN não procura voluntariamente tratamento, e outro fator importante a ser levado em conta é que a doença, em geral, tem seu início na adolescência, deixando a decisão da procura dos serviços especializados, de modo geral, para os responsáveis legais.

Examinando a questão mais profundamente, vê-se que o paciente anorético não é incompetente em todos os aspectos. A sua questão é direcionada obsessivamente para a questão do não ganho de peso. A resistência ao tratamento não significa que eles não desejem ser tratados, porém a patologia em si os leva a sentimentos de conflito entre o desejo de ficar "bem" e o medo da "gordura". Por outro lado, não pode o profissional médico assegurar a completa recuperação, pois se sabe que, mesmo em centros altamente especializados, essa chega, no máximo, a $55 \%$ a $60 \%{ }^{28}$. A técnica de tratamento é empírica porque um método de referência nesse domínio não existe ${ }^{29}$. O tratamento propõe que a recuperação do peso leve não somente à melhora física, mas psicológica, incluindo a melhora da cognição e das relações interpessoais que, geralmente, estão comprometidas. Sabe-se que a inanição compromete a atenção ${ }^{30,31}$ e que há alterações anatômicas cerebrais nos pacientes afetados pela doença, como já mencionado anteriormente.

Quando o tratamento voluntário não é aceito ou a vida do paciente está sob risco, os clínicos julgam que devem recorrer à lei para submeter o paciente com AN ao tratamento ou permitir os programas involuntários de alimentação (nutrição enteral ou parenteral) para que o peso do paciente seja recuperado ${ }^{5}$. Ramsay et al. ${ }^{32}$ encontraram que $16 \%$ dos pacientes diagnosticados como portadores de AN, freqüentemente, nos centros psiquiátricos foram submetidos ao Mental Health $\mathrm{Act}^{33}$, antes de iniciar o tratamento de maneira voluntária. De acordo com Gunn e Taylor ${ }^{34}$, é a legislação que mais afeta os indivíduos com transtornos psiquiátricos no Reino Unido. No estudo de Ramsay et al..32, tanto os pacientes interditados quanto os voluntários obtiveram bom progresso no que se referiu ao ganho de peso, apesar de ter sido mais lenta nos pacientes internados compulsoriamente e, em relação à mortalidade, essa também foi mais alta nos compulsoriamente tratados.

O internamento compulsório de pacientes com TA por meio da ação judicial é controverso. De maneira simplifica- da, algumas indicações de internamento imediato nos casos de anorexia são: risco de suicídio, síncope, perda rápida de peso, convulsões, bradicardia, distúrbios hidroeletrolíticos, hipoglicemia, ausência de resposta ao tratamento ambulatorial, ambiente familiar muito comprometido ${ }^{35}$.

No que diz respeito ao tratamento involuntário das doenças psiquiátricas em geral, as opiniões divergem, especialmente no que se refere aos TA. Assim, as opiniões entre os profissionais de saúde divergem sobre o uso da legislação para o tratamento compulsório da $\mathrm{AN}^{36}$.

As opiniões divergem sobre o uso da coerção (seja legal ou não) em relação ao tratamento. Alguns programas rejeitam totalmente a imposição ${ }^{37}$, enquanto outros vêem a alimentação nasogástrica forçada como elemento integral que pode e deve ser utilizado ${ }^{38}$. Os argumentos contra o internamento compulsório abrangem duas linhas diferentes: a possível falta de eficácia clínica e as dificuldades ético-legais. Por razões éticas, a efetividade do tratamento compulsório nessa área é difícil de ser avaliada na forma de estudos empíricos e impossível de ser realizada na forma de intervenções experimentais ou randomizadas. Não obstante controvérsia do tratamento compulsório em psiquiatria e os aspectos legais, há poucos estudos sobre a evolução dos pacientes internados com TA de maneira compulsória ${ }^{15}$. Um estudo sugeriu que o tratamento compulsório em adultos possa não ser efetivo em alcançar os efeitos desejáveis a longo prazo, apesar de a curto prazo os pacientes internados sem consentimento próprio alcancem o peso esperado ${ }^{32}$. Gowers et al. ${ }^{39}$ encontraram que a admissão compulsória de adolescentes com AN era fator preditivo de má evolução. Um estudo no sul da Austrália, no qual foram seguidos 200 pacientes com AN por cinco anos, concluiu-se que, para os casos graves, o tratamento obteve efeitos pouco relevantes, apesar de haver problemas metodológicos na avaliação 29 .

\section{“CRENÇAS" E CONCLUSÕES}

Tan, Hope e Stewart ${ }^{18}$ em um estudo qualitativo com dez pacientes do gênero feminino com o diagnóstico de AN, com idades variando entre 13 a 21 anos, tentaram avaliar as crenças e os valores das entrevistadas no que se referia à competência de recusar o tratamento. Foi utilizada entrevista semi-estruturada com enfoque no impacto da doença sobre as atitudes em relação à morte e à incapacidade, sobre os valores e a identidade pessoais e a ambivalência no que refere ao tratamento e possível recuperação. Do ponto de vista quantitativo, utilizou-se o The MacArthur Treatment Competence Study (MacArthur-T, 1995) ${ }^{40}$, que é o instrumento padrão mais completo utilizado para avaliar competência, segundo a definição da mesma adotada pela legislação da Inglaterra e dos Estados Unidos. O nível de psicopatologia foi investigado por meio do uso de cinco questionários 
e nesses (considerando-se a média de todos em conjunto) observou-se que o grupo pontuava muito acima do esperado para a população em geral. No entanto, ainda que houvesse esse provável nível de psicopatologias associadas, os resultados de MacArthur-T evidenciaram que, em geral, os participantes apresentavam bom entendimento da doença, suas conseqüências e as opções de tratamento disponíveis. Ainda que entendessem esses fatos, duas participantes não aceitaram que eram anoréticas. A análise qualitativa gerou dados muito interessantes. Alguns pacientes descreveram que a morte e a incapacidade funcional eram menos importantes do que a doença em si, ou seja, a patologia apresentava tamanho impacto sobre suas vidas que a morte causaria o fim do sofrimento. Alguns desses participantes apresentavam depressão, porém não era a maioria. Pela importância da anorexia por si mesma, aspectos como a família, a falta de desenvolvimento acadêmico e outras relações interpessoais eram sentidas como não relevantes para os entrevistados. A doença era sentida como fazendo parte "de si mesmos", de sua identidade, e não uma patologia. Havia, também, ganho secundário: "uma forma de proteção contra outros pensamentos". Sabiam ou conheciam os benefícios do tratamento, mas tomar a decisão de trataremse era difícil porque implicava o reconhecimento de que eram portadores de patologia que ligavam ao sentimento de identidade, que pensavam protegê-las e que seria, afinal, uma mudança em suas vidas de maneira radical. Esse estudo, qualitativo em essência, mesmo com número pequeno de pacientes, reflete o discurso que, muitas vezes, é ouvido na prática diária de tratar esses pacientes em um centro de transtornos alimentares. Geralmente, são garotas jovens nas quais a doença não permitiu a formação de identidade na ausência do medo de engordar, sem os comportamentos repetitivos (contar calorias, pesarem-se várias vezes ao dia, exercícios extenuantes, abuso de laxantes e diuréticos e restrição) e sem relações interpessoais com ganho independente da doença.

Dizer que o paciente que tem essas crenças é competente é aceitar que o sofrimento tenha de fazer parte de suas vidas. Isso é respeito à autonomia? Permitir que adolescentes que podem ter uma vida produtiva, se curados, continuem e cronifiquem, é ético? Aceitar a autonomia (que deveria vir entre aspas) no caso da anorexia é ser conivente com a psicopatologia e concordar que isso seja "uma escolha". Morrer não é uma decisão do anorético: morrer é uma forma de parar de sofrer. Alguns podem argumentar que esse tipo de discussão seja inútil, afinal a maior parte dos pacientes é menor de idade e legalmente não decidem suas vidas. Mas, se entendermos suas crenças, podemos redirecioná-los. Se entendermos o medo do "nada" (ou seja, a vida sem a doença), podemos tratá-los. No entanto, a recuperação necessita do ganho de peso. Fazer que um adolescente ganhe peso para tentar a retomada de sua vida é antiético?
Outro elemento de extrema importância que não deve jamais ser negligenciado é a família. Para a família, entender a doença não é questão fácil. Muitos pais dizem que seria mais compreensível "entender que ela tivesse um câncer", como se a anorexia não fosse tão ou mais grave que muitas "doenças". Uma situação comum é que essas famílias peregrinam por meses de consultórios em consultórios, ouvindo que fazer dieta "é coisa de adolescente" ou pior, vendo suas filhas serem submetidas a exames desnecessários para achar o substrato da doença: "O orgânico". Enquanto isso desmaiam na escola, ficam em casa trancafiadas e como os pais dizem: "estão estranhas". Mas, ao chegarem ao centro de referência, quando ouvem que a garota está seriamente doente, parecem negar e dizem "vamos dar uma chance. Ela vai comer". E mais interessante é a surpresa de quem as trata com essa reação. Como pode uma família aceitar que sua filha esteja morrendo por inanição, tendo acesso ao alimento? Vêem, mas não entendem. Cabe, a quem trata, esclarecer que não é "uma futilidade", "uma agressão direcionada à família especificamente", que não se sabe as causas, que não se sabe muito, mas que se tentará ajudar e que o apoio deles com a equipe médica é essencial.

O tratamento é coercitivo? Sim. O tratamento supostamente tira a autonomia do doente? Sim. Mas, a natureza da patologia exige em graus menores ou maiores o exercício de poder que não gera alegria para quem o exerce, a não ser quando ao final o paciente diz que agradece e que nunca mais quer "entrar nisso de novo".

Não se quer, nessas palavras, dizer que se pode tudo. O que se pretende dizer e esclarecer é que "ser anorético" não é uma escolha, uma decisão fundamentada em valores que se construiu segundo crenças. A doença constrói as crenças de maneira perversa e difícil de se lidar. Mas, esse "estranho" que se "apossou" como em um ritual é passível de cura e nisso constitui a nossa crença.

\section{REFERÊNCIAS}

1. American Psychiatric Association. Diagnostic and statistical manual of mental disorders. $4^{a}$ ed. Washington, DC: American Psychiatric Association; 1994.

2. Hsu LKJ. Eating disorders. New York: Guilford Press; 1990

3. Moller-Madsen S, Nystrup J, Nielsen S. Mortality in anorexia nervosa in Denmark during the period 1970-1987. Acta Psychiatr Scand. 1996;94:454-9.

4. Crisp AH, Callender JS, Halek C, Hsu LKJ. Long term mortality in anorexia nervosa. Br J Psychiatry. 1992;94:104-7.

5. Griffiths R, Russell J. Compulsory treatment of anorexia nervosa patients. In: Vandereycken W, Beumont PJ. Treating eating disorders: ethical, legal and personal issues. London UK: The Athlone Press; 1998. p. 127-50.

6. Sullivan PF. Mortality in anorexia nervosa. Am J Psychiatry. 1995;152: 1073-4.

7. Costin C. The eating disorder sourcebook: a comprehensive guide to the causes, treatment and prevention of eating disorders. Los Angeles: Lowell House; 1997.

8. Kreipe RE, Churchill BH, Strauss J. Long term outcome of adolescents with anorexia nervosa. Am J Dis Child. 1989:43:1322-7. 
9. Nussbaum MP, Shenker IR, Baird D, Saracy S. Follow up investigation of patients with anorexia nervosa. J Pediatr. 1985;106:835-40.

10. Steiner H, Mazer C, Litt IF. Compliance and outcome in anorexia nervosa. West J Med. 1990;153:133-9.

11. British Medical Association. Assessment of mental capacity: guidance for doctors and lawyers. A report of the British Medical Association and The Law Society. London. 1995. p. 65-6.

12. Tan JOA, Jones DPH. Children's consent. Curr Opin Psychiatry. 2001;14:303-7.

13. Grisso T, Appelbaum PS. Assessing competence to consent to treatment: a guide for physicians and other health professionals. Oxford: UK: Oxford University Press; 1998. p. $173-200$

14. Appelbaum PS, Rumpf T. Civil commitment of the anorexic patient. Gen Hosp Psychiatry. 1998;20(4):225-30

15. Watson TL, Bowers WA, Andersen AE. Involuntary treatment of eating disorders. Am J Psychiatry. 2000;157:1806-10.

16. Probst M, Vandereycken W, Wanderlinden J, Van Coppenolle H. The significance of body size estimation in eating disorders: its relationship with clinical and psychological variables. Int J Eat Disord. 1998;24:167-74.

17. Herpetz-Dahlmann B, Muller B, Herpetz S, Heussen N, Hebebrand J, Remshmidt H. Prospective 10-year follow-up in adolescent anorexia nervosa - course, outcome, psychiatric comorbidity, and psychosocial adaptation. J Child Psychol Psychiatry. 2001:42(5):603-12

18. Tan JOA, Hope T, Stewart A. Anorexia nervosa and personal identity: the accounts of patients and their parents. Int J Law Psychiatry. 2003;26(5):535-48.

19. Delvenne V, Goldman S, DeMaertelaer V, Lotstra F. Brain glucose metabolism in eating disorders assessed by positron emission tomography. Int J Eat Disord. 1999;25:29-37.

20. Naruo T, Nakapeppu Y, Deguchi D, Nagai N, Tsutsui J, et al. Decreases in blood perfusion of the anterior circulate gyri in anorexia nervosa restricters assessed by SPECT image analysis. BMC Psychiatry. 2001;1:2.

21. Takano A, Shiga T, Kitagawa N, Koyama T, Katoh C, et al. Abnormal neuronal network in anorexia nervosa studied with I-123-IMP SPECT. Psychiatry Res. 2001;107:45-50.

22. Wu JC, Gagman J, Buchsbaum MS, Blender D, Derrfler M, et al. Greater left cerebral hemispheric metabolism in bulimia assessed by positron emission tomography. Am J Psychiatry. 1990;147:309-12.

23. Nozoe S, Naruo T, Nakabeppu Y, Soejima Y, Nagaco M, et al. Changes in regional cerebral blood flow in patients with anorexia nervosa detecte through single photon emission tomography imaging. Biol Psychiatry. 1993;34:578-80.
24. Ellison Z, Foong J, Howard R, Bullmore E, Williams S, Treasure J. Functional anatomy of calorie fear in anorexia nervosa (Letter). Lancet. 1998;352:1152.

25. Fischer M, Golden NH, Katzman DK, Krupe RE, Schbendack J, Sigman G, et al. Eating disorders in adolescents: a background paper. J Adolesc Health. 1989;16:420-37.

26. Howard WT, Evans KK, Quitero-Howard CV, Bowers WA, Andersen AE. Predictors of success or failure of transition to day hospital treatment for inpatients with anorexia nervosa. Am J Psychiatry. 1999;156:1697-702

27. Baran SA, Weltzin TE, Kaye WH. Low discharge weight and outcome in anorexia nervosa. Am J Psychiatry. 1995;152:1070-2.

28. Steinhausen HC. The outcome of anorexia nervosa in the 20th century. Am J Psychiatry. 2002;159: 1284-93

29. Ben-Tovim D. Outcomes in patients with eating disorders: A 5-year study. Lancet. 2001;357:1254-7.

30. Strup BJ, Weingarten H, Kaye W, Gwirtsman H. Cognitive processing in anorexia nervosa: a disturbance in automatic information processing. Neuropsycobiology. 1986;15:89-94.

31. Dodin V, Nandrino JL. Cognitive processing of anorexic patients in recognition tasks: AN event-related potentials study. Int J Eat Disord. 2003;33:299-307.

32. Ramsay R, Ward A, Treasure J, Russell GMF. Compulsory treatment in anorexia nervosa. Br J Psychiatry. 1999;175:147-53.

33. Mental Health Act 1983. Department of Health-Publications. www.dh.gov.uk/en/PublicationsAndStatistics/Legislation/ActsAndBills/DH_4002034.Acessado em 22/07/2007.

34. Gunn J, Taylor PJ. Forensic psychiatry: clinical, legal \& ethical issues. Oxford: Butterworth Heinemann; 2000

35. Goldner EM, Birmingham CL. Anorexia nervosa: methods of treatment. In: Alexander-Mott L, Lumsden DB. Understanding eating disorders: anorexia nervosa, bulimia nervosa and obesity. Hove, England: Taylor \& Franas; 1994.

36. Vandereycken W, Beumont PJV, editors. Treating eating disorders: ethical, legal and personal issues. New-York: New York University Press; 1998.

37. Fost N. Food for thought: dresser on anorexia. Wis L Rev. 1984;375-84.

38. Rathner G. A plea against compulsory treatment of anorexia nervosa patients. In: Vandereycken W, Beumont PJ. Treating eating disorders: ethical, legal and personal issues. London: The Athlone Press; 1998. p. 179-215.

39. Gowers SG, Weetman J, Shore A, Houssain F, Elvins R. Impact of hospitalization on the outcome of adolescent anorexia nervosa. Br J Psychiatry. 2000;176:138-41.

40. Angela SG, Angela MP, Janelle WC, Shahana H, Nancy AH, Leslie JH. Perceived Coercion and Change in Perceived Need for Admission in Patients Hospitalized for Eating Disorders. Am J Psychiatry 2007;164(1):108-14. 